\title{
GLOBAL (AND LOCAL) ANALYTICITY FOR SECOND ORDER OPERATORS CONSTRUCTED FROM RIGID VECTOR FIELDS ON PRODUCTS OF TORI
}

\author{
DAVID S. TARTAKOFF
}

\begin{abstract}
We prove global analytic hypoellipticity on a product of tori for partial differential operators which are constructed as rigid (variable coefficient) quadratic polynomials in real vector fields satisfying the Hörmander condition and where $P$ satisfies a "maximal" estimate. We also prove an analyticity result that is local in some variables and global in others for operators whose prototype is

$$
P=\left(\frac{\partial}{\partial x_{1}}\right)^{2}+\left(\frac{\partial}{\partial x_{2}}\right)^{2}+\left(a\left(x_{1}, x_{2}\right) \frac{\partial}{\partial t}\right)^{2}
$$

(with analytic $a(x), a(0)=0$, naturally, but not identically zero). The results, because of the flexibility of the methods, generalize recent work of Cordaro and Himonas in [4] and Himonas in [8] which showed that certain operators known not to be locally analytic hypoelliptic (those of Baouendi and Goulaouic [1], Hanges and Himonas [6], and Christ [3]) were globally analytic hypoelliptic on products of tori.
\end{abstract}

\section{INTRODUCTION}

We are concerned here with the global analytic hypoellipticity of second-order operators of the form

$$
P=\sum_{j, k=1}^{m} a_{j k}(x, t) X_{j} X_{k}+\sum_{j=1}^{m} b_{j}(x, t) X_{j}+X_{0}+c(x, t)
$$

on a product of tori,

$$
\mathbb{T}^{N}=\mathbb{T}^{m} \times \mathbb{T}^{n}
$$

where $x \in \mathbb{T}^{m}, t \in \mathbb{T}^{n}$. Here the functions $a_{j k}, b_{j}$ and $c(x, t)$ may be complex valued, but the "rigid" vector fields

$$
X_{j}=\sum_{k=1}^{m} d_{j k}(x) \frac{\partial}{\partial x_{k}}+\sum_{k=1}^{n} e_{j k}(x) \frac{\partial}{\partial t_{k}}
$$

are real. The hypotheses we make are that

$$
\left\{X_{j}^{\prime}=\sum_{k=1}^{m} d_{j k}(x) \frac{\partial}{\partial x_{k}}\right\}
$$

Received by the editors November 21, 1994.

1991 Mathematics Subject Classification. Primary 32F10, 35N15, 35B65. 
are independent, $j=1, \ldots, m$, and there exists a constant $C$ such that for all smooth $v$,

$$
\sum_{j=1}^{m}\left\|X_{j} v\right\|_{L^{2}}^{2}+\|v\|_{L^{2}}^{2} \leq C\left\{\left|\Re(P v, v)_{L^{2}}\right|+\|v\|_{-1}^{2}\right\} .
$$

For example, if the vector fields $\left\{X_{j}\right\}_{j=0, \ldots, m}$ satisfy the Hörmander condition that their iterated commutators span the whole tangent space and the matrix $A=\left(a_{j k}\right)$ is the identity, then one even has a subelliptic estimate, which implies arbitrary positivity (an arbitrarily large multiple of the second term on the left, provided one adds a corresponding sufficiently large multiple of the negative norm on the right). The positivity of the self-adjoint matrix $A=\left(a_{j k}\right)$ alone will give an estimate of this form without the second term on the left and with the norm on the right replaced by the $L^{2}$ norm, but we need very slightly more. For example, the positivity of $A$ together with

$$
\begin{gathered}
-\sum\left(X_{j} a_{j k}\right) X_{k}-\sum\left(\left(X_{j} a_{j k}\right) X_{k}\right)^{*}+\sum b_{j}(x, t) X_{j} \\
+\left(\sum b_{j}(x, t) X_{j}\right)^{*}+X_{0}+X_{0}^{*}+c(x, t)>0
\end{gathered}
$$

will suffice, and this in turn would follow from sufficient positivity of the zero order term $c(x, t)$.

This class of operators generalizes that given in [4], and in our opinion simplifies the proof. The more flexible proof techniques we employed in [12] and [13] allow us to handle this broader class of operators. At one point in [4] the authors also prove a theorem of analyticity that is global in some variables and local in others for operators like

$$
p=\left(\frac{\partial}{\partial x_{1}}\right)^{2}+\left(\frac{\partial}{\partial x_{2}}\right)^{2}+\left(a(x) \frac{\partial}{\partial t}\right)^{2} .
$$

Our methods apply to these operators as well (cf. Theorems 2, 3, and 4).

Our interest in these problems was stimulated by the work of Cordaro and Himonas [4].

\section{Statement of the theorems}

Theorem 1. Let $P$ be a partial differential operator of the form (1) above with real analytic coefficients $a_{j k}(x, t), b_{k}(x, t)$, and $c(x, t)$, where the real analytic vector fields $\left\{X_{j}\right\}_{j=0, \ldots, n}$ are "rigid" in the sense of (4). Assume that $P$ satisfies the a priori estimate (5) for some $C \geq 0$. Then $P$ is globally analytic hypoelliptic; that is, if $v$ is a distribution on $\mathbb{T}^{N}$, with Pv analytic on $\mathbb{T}^{N}$, then $v$ itself is analytic on $\mathbb{T}^{N}$.

We also state three theorems which are local in some variables and global in others. In so doing, we hope to elucidate the distinction between local and global analyticity. These results are stated for rather explicit, low-dimensional operators for easy reading. For much fuller and more general results, the reader is referred to the forthcoming paper of Bove and Tartakoff ([2]). The restriction to secondorder operators is undoubtedly artificial, as the methods of our recent paper with Popivanov [10] suggest.

First we assume that $x \in \mathbb{T}^{2}$, but that $t \in I$, where $I$ is an open interval. 
Theorem 2. Let the operator $P$ be given by

$$
P=\left(\frac{\partial}{\partial x_{1}}\right)^{2}+\left(\frac{\partial}{\partial x_{2}}\right)^{2}+\left(a\left(x_{1}, x_{2}\right) \frac{\partial}{\partial t}\right)^{2}=\sum_{1}^{3} X_{j}^{2}
$$

with $x \in \mathbb{T}^{2}$ but $t \in I, I$ an interval. Then if $a\left(x_{1}, x_{2}\right)$ is analytic, zero at 0 but not identically zero (so that the Hörmander condition is satisfied for $P$ ), and $P u=f$ with $f$ real analytic on $\mathbb{T}^{2} \times I$, then $u$ is also analytic on $\mathbb{T}^{2} \times I$.

Remark. Theorem 2 holds for a wide class of operators of this type. For example, if we denote by $Y_{j}$ the vector fields

$$
Y_{1}=\frac{\partial}{\partial x_{1}}, \quad Y_{2}=\frac{\partial}{\partial x_{2}}, \quad \text { and } \quad Y_{3}=a\left(x_{1}, x_{2}\right) \frac{\partial}{\partial t},
$$

then Theorem 2 holds for any second-order polynomial in the $Y_{j}$

$$
P=\sum_{|\alpha| \leq 2} b_{\alpha}(x, t) Y_{I_{\alpha}}
$$

with (nonrigid) variable coefficients $b_{\alpha}(x, t)$ such that $(5)$ holds with $X_{j}$ replaced by $Y_{j}$.

Next we look at what happens with $x_{1} \in I_{1}, x_{2} \in \mathbb{T}^{2}$, and $t \in I_{2}$, when the coefficient $a(x)=a\left(x_{1}\right)$, where the $I_{j}$ are open intervals.

Theorem 3. Let the operator $P$ be given by

$$
P=\left(\frac{\partial}{\partial x_{1}}\right)^{2}+\left(\frac{\partial}{\partial x_{2}}\right)^{2}+\left(a\left(x_{1}\right) \frac{\partial}{\partial t}\right)^{2}=\sum_{1}^{3} X_{j}^{2}
$$

with $x_{1} \in I_{1}, x_{2} \in \mathbb{T}^{1}$, and $t \in I_{2}$, the $I_{j}$ being intervals. Then if $a\left(x_{1}\right)$ is analytic, zero at 0 but not identically zero (so that the Hörmander condition is satisfied for $P)$, and $P u=f$ with $f$ real analytic on $I_{1} \times \mathbb{T}^{1} \times I_{2}$, then $u$ is also analytic on $I_{1} \times \mathbb{T}^{1} \times I_{2}$.

Finally, we consider the case where $a(x)=a\left(x_{1}, x_{2}\right)$, not identically zero, has the form

$$
a^{2}(x)=a_{1}^{2}\left(x_{1}\right)+a_{2}^{2}\left(x_{2}\right) .
$$

Theorem 4. Let the operator $P$ be given by

$$
P=\left(\frac{\partial}{\partial x_{1}}\right)^{2}+\left(\frac{\partial}{\partial x_{2}}\right)^{2}+\left(a_{1}^{2}\left(x_{1}\right)+a_{2}^{2}\left(x_{2}\right)\right)\left(\frac{\partial}{\partial t}\right)^{2}=\sum_{1}^{4} X_{j}^{2}
$$

with $x \in \mathbb{T}^{m}$ but $t \in I, I$ an interval. Then if $a(x)$ is analytic, zero at 0 but not identically zero (so that the Hörmander condition is satisfied for $P$ ), and $P u=f$ with $f$ real analytic near 0 , then $u$ is real analytic near 0 .

Remark. These theorems have evident microlocal versions and allow suitable variable coefficient combinations of the appropriate vector fields as well as the addition of lower-order terms in these vector fields $\left(\frac{\partial}{\partial x_{1}}, \frac{\partial}{\partial x_{2}}\right.$, and $a(x) \frac{\partial}{\partial t}$ for Theorem 2; $a_{1}\left(x_{1}\right) \frac{\partial}{\partial t}$ for Theorem 3 ; and $a_{1}\left(x_{1}\right) \frac{\partial}{\partial t}$ and $a_{2}(x) \frac{\partial}{\partial t}$ and $a_{2}\left(x_{2}\right) \frac{\partial}{\partial t}$ for Theorem 4). 


\section{Proofs of the theOREMS}

For the moment we shall assume that $v$ and $u$ are known to belong to $C^{\infty}$, and at the end we make some comments about the $C^{\infty}$ regularity of the solutions.

3.1. Proof of Theorem 1. Using well-known results, it suffices to show that in the $L^{2}$ norm we have Cauchy estimates on derivatives of $v$ of the form

$$
\left\|X^{\alpha} T^{\beta} v\right\|_{L^{2}} \leq C^{|\alpha|+|\beta|+1}|\alpha| !|\beta| !
$$

for all $\alpha$ and $\beta$. And microlocally, since the operator is elliptic in the complement of the span $W$ of the vector fields $\frac{\partial}{\partial t_{j}}$, it suffices to look near $W$; there all derivatives are bounded by powers of the $\frac{\partial}{\partial t_{j}}$ alone. That is, modulo analytic errors, we may take $\alpha=0$ and indeed $\beta=(0, \ldots, 0, r, 0, \ldots, 0)$, as follows by integration by parts and a simple induction. Here $T=\left(T_{1}, \ldots, T_{m}\right)$ with

$$
T_{k}=\frac{\partial}{\partial t_{k}}
$$

$k=1, \ldots, m$, and we shall take $T^{\beta}=T_{1}^{b}$ for simplicity. In particular, note that

$$
\left[T_{l}, P\right]=\sum_{j, k=1}^{m} a_{j k}^{\prime}(x, t) X_{j} X_{k}+\sum_{j=1}^{m} b_{j}^{\prime}(x, t) X_{j}+c^{\prime}(x, t)
$$

and thus

$$
\begin{aligned}
\left|\Re\left(\left[P, T_{1}^{b}\right] v, T_{1}^{b} v\right)_{L_{2}}\right| & \leq C\left|\sum_{b^{\prime} \geq 1}\left(\begin{array}{c}
b \\
b^{\prime}
\end{array}\right)\left(c^{\left(b^{\prime}\right)} X^{2} T_{1}^{b-b^{\prime}} v, T^{b} v\right)_{L^{2}}\right| \\
& \leq\left|\sum_{b^{\prime} \geq 1}\left(\begin{array}{c}
b \\
b^{\prime}
\end{array}\right)\left(c^{\left(b^{\prime}+1\right)} X T_{1}^{b-b^{\prime}} v,(X) T_{1}^{b} v\right)_{L^{2}}\right| \\
& \leq \text { l.c. } \sum_{b^{\prime} \geq 1} b^{2 b^{\prime}} C_{c}^{2\left(b^{\prime}+1\right)}\left\|X T_{1}^{b-b^{\prime}} v\right\|_{L^{2}}^{2}+\text { s.c. }\left\|(X) T_{1}^{b} v\right\|_{L^{2}}^{2},
\end{aligned}
$$

where the $(X)$ on the right represents the fact that this $X$ may not or may be present, depending on whether $c^{\left(b^{\prime}\right)}$ received one more derivative. We have written $X^{2}$ for a generic $X_{j} X_{k}$ as well as $C_{c}$ for the largest of the constants which appear in the Cauchy estimates for the analytic coefficients in $P$. The large constant (l.c.) and small constant (s.c.) are independent of $b$, of course, the small constant being small enough to allow this term to be absorbed on the left-hand side of the inequality. Absorbing yields:

$$
\begin{aligned}
\sum_{1}^{m} \| & X_{j} T_{1}^{b} v\left\|_{L^{2}}^{2}+\right\| T_{1}^{b} v \|_{L^{2}}^{2} \\
& \leq C\left\{\left\|T^{b} P v\right\|_{L^{2}}^{2}+\sum_{b \geq b^{\prime} \geq 1} b^{2 b^{\prime}} C_{c}^{2\left(b^{\prime}+1\right)}\left\|X T_{1}^{b-b^{\prime}} v\right\|_{L^{2}}^{2}\right\}
\end{aligned}
$$

which, iterated until the last term on the right is missing, gives

$$
\sum_{1}^{n}\left\|X_{j} T_{1}^{b} v_{2}\right\|_{L^{2}}^{2}+\left\|T_{1}^{b} v\right\|_{L^{2}}^{2} \leq C_{(P v)}^{b+1} b !,
$$

which implies the analyticity of $v$. 
Finally, we have taken the solution to belong to $C^{\infty}$; for the $C^{\infty}$ behavior of the solution, the methods of [11] which utilize the hypoellipticity techniques of [9] will lead quickly to the $C^{\infty}$ result.

3.2. Proof of Theorem 2. We recall that we are (for simplicity) taking the operator $P$ to have the particular form

$$
P=\left(\frac{\partial}{\partial x_{1}}\right)^{2}+\left(\frac{\partial}{\partial x_{2}}\right)^{2}+\left(a(x) \frac{\partial}{\partial t}\right)^{2} .
$$

Again we take $u \in C^{\infty}$, since subellipticity is a local phenomenon and the operators we are dealing with are clearly subelliptic under our hypotheses. And again it suffices to estimate derivatives in $L^{2}$ norm, i.e., to show that with $\phi, \psi$ of compact support, and

$$
X_{j}=\frac{\partial}{\partial x_{1}}, \frac{\partial}{\partial x_{2}}, \text { or } a(x) \frac{\partial}{\partial t}
$$

we will have

$$
\sum_{j}\left\|X_{j} \psi(x) \phi(t) Z^{p} u\right\|_{L^{2}} \leq C_{u}^{p+1} p !
$$

where each $Z$ is (also) of the form $Z=\frac{\partial}{\partial x_{1}}, \frac{\partial}{\partial x_{2}}$, or $a(x) \frac{\partial}{\partial t}$. That this will suffice is due to a result by Helffer and Mattera ([7]), but it will not save us any work as we find ([12], [13]) that in trying to bound powers of the first two types of $Z$ we need to establish analytic type growth of derivatives measured by powers of $Z$ of the form $\frac{\partial}{\partial t}$ itself. Actually, we shall show that for any given $N$, there exists a localizing function $\phi_{N}(t) \in C_{0}^{\infty}$ and $\psi(x)$ (independent of $N$ ) with

$$
\sum_{j}\left\|X_{j} \psi(x) \phi_{N}(t) Z^{p} u\right\|_{L^{2}} \leq C_{u}^{p+1} N^{p}, \quad p \leq N .
$$

And, in fact, the functions $\phi_{N}(t)$ will be chosen to satisfy

$$
\left|D^{r} \phi_{N}(t)\right| \leq C_{u}^{r+1} N^{r}, \quad r \leq N,
$$

uniformly in $N$.

The philosophy of all $L^{2}$ proofs is to replace $v$ in (5) by $\psi(x) \phi(t) \widetilde{Z}^{p} u$ with $\widetilde{Z}=\frac{\partial}{\partial x_{1}}, \frac{\partial}{\partial x_{2}}$, or $\frac{\partial}{\partial t}$, and commute $\psi(x) \phi(t) \widetilde{Z}^{p}$ past the differential operator $P$. For argument's sake, and since everything else is simpler, we may restrict ourselves to the worst case, which is given by $\widetilde{Z}=\frac{\partial}{\partial t}$. In so doing, we encounter the errors

$$
\left[\frac{\partial}{\partial x_{1}}, \psi \phi\left(\frac{\partial}{\partial t}\right)^{p}\right], \quad\left[\frac{\partial}{\partial x_{2}}, \psi \phi\left(\frac{\partial}{\partial t}\right)^{p}\right], \quad \text { and } \quad\left[a(x) \frac{\partial}{\partial t}, \psi \phi\left(\frac{\partial}{\partial t}\right)^{p}\right] .
$$

Thus, starting with a given value of $p$, the left-hand side of the a priori inequality (5) will bound $X_{j} \psi(x) \phi(t)\left(\frac{\partial}{\partial t}\right)^{p} u$ in $L^{2}$ norm (after taking the inner product on the right and integrating by parts one derivative to the right) by

$$
\left\|\frac{\partial \psi(x) \phi(t)}{\partial x}\left(\frac{\partial}{\partial t}\right)^{p} u\right\|_{L^{2}} \text { and }\left\|a(x) \frac{\partial \psi(x) \phi(t)}{\partial t}\left(\frac{\partial}{\partial t}\right)^{p} u\right\|_{L^{2}}
$$

(and related terms arising from the integration by parts, terms which exhibit the same qualitative behavior as these). 
So, at the very least, we have bounded $\left\|X_{j} \psi(x) \phi(t)\left(\frac{\partial}{\partial t}\right)^{p} u\right\|_{L^{2}}$ by

$$
\left\|\psi^{\prime}(x) \phi(t)\left(\frac{\partial}{\partial t}\right)^{p} u\right\|_{L^{2}} \text { and }\left\|a(x) \psi(x) \phi^{\prime}(t)\left(\frac{\partial}{\partial t}\right)^{p} u\right\|_{L^{2}} .
$$

In the first term, we have lost the "good" derivative $\frac{\partial}{\partial x}$ and seen it appear on the localizing function, but we cannot iterate this process, since the a priori estimate (5) is only truly effective when a "good" derivative is preserved. When no "good" derivative is preserved, we have seen often enough that only in the global situation when the derivative that appeared on the localizing function can be absorbed by a constant by the introduction of a partition of unity (in that variable, in this case it is the $x$, or toroidal, variable), can one obtain analyticity (in that variable). The second type of term in (23) is actually good, since the factor $a(x)$ will combine with one of the "bad" derivatives $\frac{\partial}{\partial t}$ to give a "good" derivative $Z=a(x) \frac{\partial}{\partial t}$, which may be iterated under (5). That is, modulo terms which lead to global analyticity in $x$, we have the iteration schema

$$
\sum_{j}\left\|X_{j} \psi(x) \phi(t)\left(\frac{\partial}{\partial t}\right)^{p} u\right\|_{L^{2}} \rightarrow C \sum_{j}\left\|X_{j} \psi(x) \phi^{\prime}(t)\left(\frac{\partial}{\partial t}\right)^{p-1} u\right\|_{L^{2}},
$$

which may indeed be iterated. The result of multiple iterations is, for $\phi(t)=\phi_{N}(t)$ satisfying the estimates (16) but the localizations in $x$ merely smooth and subject to $\sum_{k} \psi_{k}(x)=1$ (none will ever receive more than a couple of derivatives),

$$
\begin{aligned}
\sum_{j, k} \| & X_{j} \psi_{k}(x) \phi_{N}(t)\left(\frac{\partial}{\partial t}\right)^{p} u \|_{L^{2}} \\
\leq & \sum_{\substack{j, k \\
p^{\prime} \leq p}} C^{p^{\prime}}\left\|\psi_{k}(x) \phi_{N}^{\left(p-p^{\prime}\right)}(t)\left(\frac{\partial}{\partial t}\right)^{p^{\prime}} P u\right\|_{L^{2}} \\
& +\sum_{\substack{j, k \\
p^{\prime} \leq p}} C^{p} N^{p^{\prime}}\left\|\left(X_{j}\right) \psi_{k}(x) \phi_{N}^{\left(p^{\prime}\right)}(t) u\right\|_{L^{2}} .
\end{aligned}
$$

Since $p \leq N$ and $N^{N} \leq C^{N+1} N$ ! by Stirling's formula, under the bounds (16), this yields the desired analyticity, which is local in $t$ but global in $x$.

3.3. Proof of Theorem 3. The new ingredient in Theorem 3 is that the function $a(x)$ is now of a more special form. Thus it is only on the hypersurface $x_{1}=0$ that the operator $P$ is not elliptic. If in the above proof we replace the compactly supported function $\psi(x)$ by a product

$$
\psi(x)=\psi_{1}\left(x_{1}\right) \psi_{2}\left(x_{2}\right),
$$

with both $\psi_{j}(s)$ equal to one near $s=0$, then when derivatives enter on $\psi_{1}\left(x_{1}\right)$, the support of $\psi_{1}^{\prime}$ is contained in the elliptic region, and only in $x_{2}$ does one need to pass to further and further patches, ultimately using a (finite) partition of unity on the torus in $x_{2}$.

3.4. Proof of Theorem 4. The new ingredient in Theorem 4 is that there are four vector fields:

$$
\frac{\partial}{\partial x_{1}}, \quad \frac{\partial}{\partial x_{2}}, \quad a_{1}\left(x_{1}\right) \frac{\partial}{\partial t}, \quad \text { and } \quad a_{2}\left(x_{2}\right) \frac{\partial}{\partial t} .
$$


The above considerations apply to $x_{1}$ and $x_{2}$ separately now, since if either $x_{1} \neq 0$ or $x_{2} \neq 0$ we are in the elliptic region where the solution is known to be analytic.

Remark. It is not hard to see that derivatives in $x_{1}$ and $x_{2}$ always behave well; i.e., that $\left(x_{1}, x_{2}, t ; \xi_{1}, \xi_{2}, \tau\right)$ is never in the analytic wave front set $W F_{A}(u)$ for $\left(\xi_{1}, \xi_{2}\right) \neq(0,0)$ whenever this is true (punctually) of $P u$, since only points of the form $\left(x_{1}, x_{2}, t ; 0,0, \tau\right)$ are characteristic for $P$. Hence, the above theorems are actually "microlocal(-global)" in a sense which is fairly evident, much as in [5].

\section{REFERENCES}

1. M. S. Baouendi and C. Goulaouic, Analyticity for degenerate elliptic equations and applications, Proc. Sympos. Pure Math., vol. 23, Amer. Math. Soc., Providence, RI, 1971, pp. 79-84. MR 50:5167

2. A. Bove and D. S. Tartakoff, Microlocal Gevrey hypoellipticity for subelliptic operators, (to appear).

3. M. Christ, Certain sums of squares of vector fields fail to be analytic hypoelliptic, Comm. Partial Differential Equations 10 (1991), 1695-1707. MR 92k:35056

4. P. Cordaro and A. Himonas, Global analytic hypoellipticity of a class of degenerate elliptic operators on the torus, Math. Res. Lett. 1 (1994), 501-510. MR 95j:05048

5. M. Derridj and D. S. Tartakoff, Global analyticity for $\square_{b}$ on three dimensional pseudoconvex CR manifolds, Comm. Partial Differential Equations 18 (11) 1993, 1847-1868. MR 94i:32021

6. N. Hanges and A. Himonas, Singular solutions for sums of squares of vector fields, Comm. Partial Differential Equations 16 (1991), 1503-1511. MR 92i:35031

7. B. Helffer and C. Mattera, Analyticité de itérés réduits d'un système de champs de vecteurs, Comm. Partial Differential Equations 5 (1980), 1065-1072. MR 81m:35034

8. A. Alexandrou Himonas, On degenerate elliptic operators of infinite type, Math. Z. (to appear).

9. L. Hormander, Linear partial differential operators, Springer-Verlag, New York, 1969. MR 40:1687

10. P. Popivanov and D. S. Tartakoff, Gevrey hypoellipticity for fourth order differential operators, Comm. Partial Differential Equations 20 (1995), 309-314. MR 95i:35056

11. D. S. Tartakoff, Gevrey hypoellipticity for subelliptic boundary value problems, Comm. Pure Appl. Math. 26 (1973), 251-312. MR 49:7586

12. D. S. Tartakoff, On the global real analyticity of solutions to $\square_{b}$ on compact manifolds, Comm. Partial Differential Equations 1 (1976), 283-311. MR 53:14552

13. D. S. Tartakoff, Local analytic hypoellipticity for $\square_{b}$ on nondegenerate Cauchy Riemann manifolds, Proc. Nat. Acad. Sci. U.S.A. 75 (1978), 3027-3028. MR 80g:58045

Department of Mathematics, University of Illinois at Chicago, 851 S. Morgan St., M/C 349, ChicaGo, ILlinois 60607-7045

E-mail address: dst@uic.edu 